INNOVATIONS IN PRIMARY CARE

Pharmacist-Led Chronic Care Management Services

Wei C. Yuet, PharmD

Jessica Gardea, PharmD ${ }^{1}$

Didi Ebert, DO, MPH, MS²

Randy D. Martin, PharmD ${ }^{1}$

'Department of Pharmacotherapy, University of North Texas Health Science Center, Fort Worth, Texas

${ }^{2}$ Department of Family Medicine, University of North Texas Health Science Center, Fort Worth, Texas

Ann Fam Med 2019;17:465. https://doi.org/10.1370/afm.2440.

\section{THE INNOVATION}

Chronic care management is a fee-based service that reimburses practices for enhanced services provided by clinical staff, such as care coordination and medication management for Medicare beneficiaries with 2 or more chronic diseases. ${ }^{1}$ Recent changes to chronic care management structure and reimbursement prompted our practice to implement a pharmacist-led chronic care management service to augment physician revenue and capacity. This service is provided in collaboration with physicians and other health care practitioners.

\section{WHO AND WHERE}

The University of North Texas (UNT) Health Clinical Practice Group consists of 42 physicians who provide primary and specialty care for 37,000 patients, including over 13,000 Medicare beneficiaries. Six pharmacists with specialized training are employed by the practice to provide medication management under a collaborative practice agreement with physicians.

The pharmacists are authorized to prescribe, modify, and discontinue medications; order tests; and conduct basic physical assessment.

\section{HOW}

In 2017, challenged by leadership to develop a sustainable practice model for clinical pharmacists, the Clinical Practice Group evaluated various models and determined chronic care management was best aligned with the practice's population and goals.

Conflicts of interest: autbors report none.

Corresponding author

Wei C. Yuet, PharmD

Department of Pharmacotherapy

3500 Camp Bowie Blvd

University of North Texas Health Science Center

Fort Worth, Texas 76107

cheng.yuet@unthsc.edu
Although pilot studies of pharmacist-led chronic care management have been described, these studies occurred before changes to chronic care management program rules and reimbursement implemented in 2017.1-3 Consequently, these studies failed to demonstrate cost effectiveness, leading many practices to deliver chronic care management via less expensive clinical staff (ie, registered or licensed vocational nurses). ${ }^{4}$ Important program changes that led our practice to choose chronic care management as a vehicle for clinical pharmacy services included: (1) expansion of Current Procedural Terminology (CPT) pertaining to chronic care management (Supplemental Table); (2) incentives for physician participation in the comprehensive care plan; and (3) considerations for moderate to complex medical decision making. It is important to note that collaborative practice laws in our state permit pharmacists to engage in medical decision making under physician supervision.

Patients are enrolled in our program during an initial pharmacist-physician co-care visit which is reimbursed by adding a G-modifier to standard coding (Supplemental Table). During this visit, the pharmacist, physician, and patient agree upon a comprehensive care plan which serves as a blueprint for future pharmacist encounters. Subsequent pharmacist visits, either via telephone or face-to-face, may be reimbursed, provided these services do not occur on the same day as a visit with another clinician and are pertinent to the comprehensive care plan. For issues beyond the scope of the pharmacist, the patient is referred to their physician or other suitable professional.

Revenue cycle management for this service was challenging because chronic care management is reimbursed based upon patient complexity and accumulated time spent each month. Our practice committed significant time and resources to build processes and templates in the electronic health record to facilitate revenue cycle processes.

\section{LEARNING}

Pharmacists partnering with physicians in primary care improve patient outcomes; however, sustainable models are limited. ${ }^{5}$ Pharmacist-led chronic care management services with a focus on complex patients can be implemented in a manner that is complementary, rather than competitive, to services provided by physicians and advanced practice clinicians, allowing physician practices to generate revenue from their activities. This program may improve access to care and the capacity to manage chronic diseases. However, significant investment may be required to develop the necessary infrastructure to support the program.

For additional information, including key words, references and Supplemental Table, see it online at http://www. AnnFamMed. org/content/17/5/465/suppI/DC1/. 